\title{
中国种堂
}

\section{大气边界层中浮力作用引起 的混合层发展过程}

\author{
肖经纬周明显 \\ (中国科学院大气物理研究所, 北京)
}

拉 要

本文利用三层模型，讨论了由浮力作用引起的混合层的形成、发展和覆盖逆温层 的抬升问题，并进一步讨论了大气边界层中基本的引过程，通过讨论能量收支问 题，建立了较合适的参数化方程. 对要盖逆温层的形成、维持及其随时间的变化作了 理论分析. 在此基础上, 还建立了描写混合层高度、逆温层厚度及其逆温强度的方 程组，并获得了相应的近似解析解。文中采用大气物理研究所的声雷达探测资料和 Wangara 实验数据检验了理论结果，计算值与观测值基本一致。

\section{一、引 言}

当太阳对地表面的加热作用超过地表本身的辐射热损失时, 夜间形成的辐射逆温层自下 而上耗散,混合层高度逐渐增加。

Ball $^{[1]}$ ，Carson ${ }^{[2]}$, Tennekes ${ }^{[3]}$ 等利用跳跃模式，Mahrt ${ }^{[4]}$ 等利用三层模式，Deardorff ${ }^{[5,6]}$ 利 用实验方法和数值模拟，吕乃平 ${ }^{[7]}$ 等利用声雷达探测对浮力作用下的混合层进行过研究. 但 是, 有关覆盖逆温层形成和维持的机理及其动力和热力特性目前尚无系统的理论研究. 已建 立的有关参数化方程 (见文献 [3]) 也不严格. 这就影响对漉合层发展中所涉及的较复杂的物 理过程作进一步的讨论.

本文的目的是对浮力作用引起的混合层的发展, 逆温层基底处发生的牵引与穿透过程作 进一步的讨论, 并妇求混合层高度、逆温层厚度及其逆温强度与初始条件和能量输人过程的关 系. 本文采用三层模式,其特点可表示为:

$$
\begin{aligned}
& \frac{\partial \Theta_{m}}{\partial z}=0, \quad \text { 于 } z \leqslant h ; \frac{\partial U_{m}}{\partial z}=0 \text {, 于 } z_{*}<z \leqslant h ; \\
& \frac{\partial^{2} \Theta_{i}}{\partial z^{2}}=0, \frac{\partial^{2} \overline{(w \theta)_{i}}}{\partial z^{2}}=0, \frac{\partial^{2} \bar{e}_{t}}{\partial z^{2}}=0 \text {, 于 } h<z \leqslant h+\Delta h \text {; } \\
& \overline{(u \cdot \theta)_{s}}=0, \bar{c}_{s}=0 \text {, } \\
& \text { 于 } z \geqslant h+\Delta h ;
\end{aligned}
$$

其中 $\Theta, U$ 和 $\bar{c}$ 分别表示平均位温、平均风速和单位质量平均湍流能量. $w$ 是速度在垂直方向 分量的脉动, $\theta$ 是位温脉动量. $h, \Delta h, z_{*}$ 分别是混合层高度、逆温层厚度和近地层高度. 下 
标 $m, i, s$ 分别表示混合层、逆温层和逆温层外的区域.

\section{二、方程组的建立与讨论}

设空气的位温是保守量,对水平均匀的湍流场,热量守恒方程是

$$
\frac{\partial \Theta}{\partial t}+W \frac{\partial \Theta}{\partial z}=-\frac{\partial}{\partial z} \overline{w \theta},
$$

式中 $W$ 是大尺度运动引起的平均垂直速度. 在混合层中, 上式变为:

$$
\frac{\partial \Theta_{m}}{\partial t}=-\frac{\partial}{\partial z} \overline{w \theta}, \quad z \leqslant h \text {. }
$$

由（1）和（2）式,有

$$
\frac{\dot{\partial}^{\prime}}{\partial z^{2}} \overline{w \theta}=0 . \quad z \leqslant h
$$

从 $z=0$ 到 $z=h$ 积分方程 (5), 给出

$$
\frac{\partial \Theta_{m}}{\partial t}=\left[\overline{(w \theta)_{0}}-\overline{(w \theta)_{h}}\right] h^{-1}, \quad z \leqslant h .
$$

对区域 $z \geqslant h+\Delta h,(4)$ 式变为:

$$
\frac{\partial \Theta_{s}}{\partial t}+W \frac{\partial \Theta_{s}}{\partial z}=0
$$

观测表明,在 $1-2$ 公里高度内, $\frac{\partial \Theta_{s}}{\partial z}$ 至少可当作分段常数处理. ${ }^{[1]}$, 因此在求解 ( 8 )式时,可设 $\Gamma=\frac{\partial \Theta}{\partial z}$ 与 $z$ 无关. 于是方程 $(8)$ 的解为:

$$
W(z)=-B z, \Gamma(t)=\Gamma(0) \exp B t,
$$

式中 $B$ 是常数, 依天气条件而定. 一般, 在 $T$ 与 $z$ 有关时仍近似有 $W=-B z^{[8]}$.

为了引入关于 $h$ 的微分方程, 利用莱卜尼兹关于交换微积运算顺序的法则, 从 $z=h$ 到 $z=h+\Delta h$ 积分 ( 4 )式,注意利用 (1)-(3) 和 (9) 式,有

$$
\begin{aligned}
-\overline{(n \theta)_{h}}= & {\left[\frac{d h}{d t}-W(h)\right] \Delta \Theta+\Theta_{h+\Delta h} \frac{d \Delta h}{d t}-\left(\frac{d}{d t}+B\right) } \\
& \times\left[\left(\frac{1}{2} \Delta \Theta+\Theta_{m}\right) \Delta h\right]+\Theta_{h+\Delta h} B \Delta h,
\end{aligned}
$$

式中 $\Delta \Theta=\Theta_{h+\Delta h}-\Theta_{m}$. 我们考虑它随时间的变化,利用 $(1),(2),(7)$ 和 (8) 式,有

$$
\begin{aligned}
\frac{d \Delta \Theta}{d t}= & {\left[\frac{d h}{d t}-W(h)\right] \Gamma(h+\Delta h, t)-\left[\overline{(t \nu \theta)_{0}}-\overline{(w \theta)_{h}}\right] h^{-1} } \\
& +\left[\left(\frac{d}{d t}+B\right) \Delta h\right] \Gamma(h+\Delta h, t) .
\end{aligned}
$$

(10)和 (11) 式描述了 $\Delta \Theta$ 和 $h$ 的变化及其相互关系, 为使它们封闭必须讨论由于热量输人低 层大气引起的湍流能量分布.

湍流能量收支方程是:

$$
\frac{\partial \bar{e}}{\partial t}=-\frac{\partial}{\partial z}\left[\overline{e w}+\frac{1}{\rho} \overline{p w}\right]-\overline{u w} \frac{\partial U}{\partial z}+\beta \overline{u^{\prime} \theta}-\varepsilon_{t},
$$


其中 $\rho$ 是密度, $P$ 是压力脉动, $\beta=\frac{g}{\theta}$ 是浮力因子, $\varepsilon_{t}$ 是单位质量湍能耗散速率. 在混合层 内部，上式可近似为：

$$
0=-\frac{\partial}{\partial z}\left[\overline{e w}+\frac{1}{\rho} \overline{p w}\right]-\overline{u w} \frac{\partial U}{\partial z}+\beta \overline{w \theta}-\varepsilon_{\prime} .
$$

在混合层顶部, 若混合层高度增加较慢, (13) 式仍适用. 若混合层迅速发展, 则 $\frac{\partial}{\partial t} \bar{e}$ 不能忽 略, 此时可考虑固定于逆温层基底上的动坐标系 $\left(x^{\prime}, y^{\prime}, z^{\prime}\right)$, 坐标变换公式是:

$$
z^{\prime}=z-t \frac{d h}{d t}
$$

微分上式,近似到一阶导数

$$
\frac{\partial z^{\prime}}{\partial t}=0-t \frac{d^{2} h}{d t^{2}}-\frac{d h}{d t} \simeq-\frac{d h}{d t} .
$$

由 (2) 和 (3) 式可知, $\frac{\partial \bar{e}}{\partial z^{\prime}}=-\frac{\bar{e}}{\Delta h}$, 于是

$$
\frac{\partial \bar{e}}{\partial t}=\frac{\partial \bar{e}}{\partial z^{\prime}} \frac{\partial z^{\prime}}{\partial t}=C_{T} \frac{\bar{e}}{\Delta h} \frac{d h}{d t},
$$

式中 $C_{T}$ 是常数,用以调整推导过程所作近似带来的影响.

下面讨论混合层中的能量分布. 从 $z=0$ 到 $z=h$ 积分方程 (13),

$$
0=-\int_{0}^{h} \frac{\partial}{\partial z}\left[\overline{e u^{\prime}}+\frac{1}{\rho} \overline{p w}\right] d z-\int_{0}^{h} \overline{u w} \frac{\partial U}{\partial z} d z+\beta \int_{0}^{h} \overline{(w \theta)} d z-\int_{0}^{h} \varepsilon_{t} d z .
$$

在近地层内可认为摩擦速度 $u_{*}$ 不随高度变化

$$
-\int_{0}^{h} \overline{u w} \frac{\partial U}{\partial z} d z=-\int_{0}^{*} \frac{\overline{u w}}{\partial z} \frac{\partial U}{\partial z} d z=u_{*}^{2} U_{m}
$$

根据 (6) 式, 并注意到在 $z=h$ 处, $\overline{(w \theta)_{h}}<0$, 有

$$
\int_{0}^{h} \overline{w \theta} d z=\frac{1}{2}\left[\overline{(w \theta)_{0}}+\overline{(w \theta)_{h}}\right] h .
$$

若混合层中的特征速度为 $u_{c}$, 则浮力生成的湍能耗散率为 $C_{t b} \frac{u_{c}^{3}}{h}, C_{t b}$ 是常数. 在充分混合的 区域,这个速率近似与高度无关 ${ }^{93}$. 在混合层中具有向下热通量的部分, 湍能主要用于牵引过 程, 能耗散可忽略. 在近地层中可认为由动力作用生成的湍能其生成速率与耗散速率成正比, 比例常数记为 $C_{t m}$, 于是

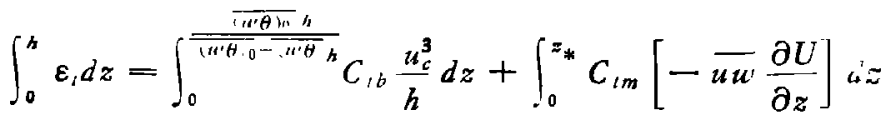

$$
\begin{aligned}
& =C_{t b} \frac{\overline{(u \theta)_{0}} u_{\cdot}^{3}}{\overline{(u \cdot \theta)_{0}-\cdots \overline{(u t \cdot \theta)_{n}}}}+C_{t m} u_{*}^{2} U_{m} .
\end{aligned}
$$

此外, 除混合层发展的最初阶段, 从它的边界流失的湍能较小，或者可略去，或者可并入 $C_{t b}$ 中, 即 


$$
\int_{0}^{h} \frac{\partial}{\partial z}\left[\overline{e w}+\frac{1}{\rho} \overline{p w}\right] d z \simeq 0
$$

将 $(15 b)-(15 e)$ 式代人 (15a) 式,给出

$$
\frac{1}{2} \frac{g}{\theta}\left[\overline{(w \theta)_{0}}+\overline{(w \theta)_{h}}\right] h-C_{t b} \frac{\overline{\left(u^{\prime}\right)_{0}} u_{c}^{3}}{(w \theta)_{0}-\overline{(u \theta)_{h}}}+\left(1-C_{t m}\right) u_{*}^{2} U_{m}=0 .
$$

利用上式可从动力学角度定义自由对流速度尺度. 对理想的、稳定的自由对流状态， $u_{*}, U_{m}$ 和 $\overline{(w \bar{\theta})_{h}}$ 均趋于零. 用 $u_{f}$ 表示对应这种状态的特征速度,则 (15) 式给出, 除一常数因子外，

$$
u_{j}=\lim _{u_{*}, v_{m},(\omega \theta)_{h} \rightarrow 0} u_{c}=\left[\frac{g}{\theta} \overline{(\omega \theta)_{0}} h\right]^{\frac{1}{2}} \text {. }
$$

在混合层发展过程中，下垫面上的边界条件变化的时间尺度是 $\overline{(w \theta)_{0}} / \frac{d \overline{(w \theta)_{0}}}{d t}$. 在后面选用 的实例中,这个尺度在北京时间 08:00 时约为 1 小时, 到 10:00 时大于 4 小时, 而泿合层中最 大的涡的特征时间 $\left(h / u_{j}\right)$ 的中等大小的值是 500 秒. 可以期望, 所讨论的过程是准稳定的, 因而可近似用 $u_{i}$ 作为速度尺度. 果若如此, 则 (15) 式变为:

$$
\frac{1}{2} \frac{g h}{\theta}\left[\frac{\left(1-2 C_{i b}\right) \overline{(w \theta)_{0}^{2}}-\overline{(w \theta)_{h}^{2}}}{(w \theta)_{0}}-\overline{(w \theta)_{h}}\right]+\left(1-C_{t m}\right) u_{*}^{2} U_{m}=0 .
$$

将 (7)式代人上式, 并注意到我们所讨论的是准稳定过程, $\frac{\partial \boldsymbol{\theta}_{m}}{\partial t}$ 和 $u_{*}^{2} U_{m}$ 均可视为常数（事实 上, $\frac{\partial \Theta_{m}}{\partial t}$ 的典型值为 $0.3 \times 10^{-30} \mathrm{~K} /$ 秒, 在 500 秒内这个数值无明显变化. 同理 $u_{*}^{2} U_{m}$ 亦可看作 常数), 则由上式看出 $\overline{(w \theta)_{0}}$ 与 $\overline{(w \theta)_{h}}$ 是双曲线型关系, 其渐近线是

$$
-(\overline{w \theta})_{h} /(\overline{w \theta})_{0}=\sqrt{1-2 C_{t b}} \text {. }
$$

它表明, 随着 $\overline{(w \theta)_{0}}$ 的增大, $-(\overline{w \theta})_{h} /\left(\overline{(w \theta)_{0}}\right.$ 趋于一常数.

顺便指出, 若 $\overline{(w \theta)_{0}} \equiv 0$, 则 $(17)$ 式给出

$$
-\overline{(w \theta)_{h}}=\frac{2\left(1-C_{t m}\right) \Theta}{g} \frac{u_{*}^{2} U_{m}}{h} .
$$

现在我们要对逆温层作一些讨论,并建立描写 $\Delta h$ 与 $h, \Delta \Theta$ 等量之间关系的方程.

逆温层的精细结构是湍流量与背景场相互作用的结果. 由混合层送来的湍流能量一部分 用来维持湍流状态,最后被耗散掉,这部分能量可表示为 $\varepsilon_{t i} \propto \frac{u_{f}^{3}}{\Delta h}$; 一部分可能用来产生重力 波. 在温度梯度较大的部分可能产生界面波,而在其上部可能产生内波,内波将以波辐射的形 式将能量传入较上层的空气。这一能轮散由 $-\frac{\partial}{\partial z}\left[\frac{1}{\rho} \overline{p w}\right]$ 决定,由因次分析有 $-\frac{\partial}{\partial z}\left[\frac{1}{\rho}\right.$. $\overline{p w}] \propto \frac{u_{L}^{3}}{\Delta h}$ (第三节将说明它的合理性). 根据这些讨论和(14)式得到逆温层基底处的能量收 支关系

$$
C_{T} \frac{u_{f}^{2}}{\Delta h} \cdot \frac{d h}{d t}=\frac{g}{\Theta} \overline{(u \cdot \theta)_{h}}+C_{F} \frac{u_{f}^{3}}{h}-C_{i} \frac{u_{f}^{3}}{\Delta h},
$$


式中已略去机械项. $C_{T}, C_{F}, C_{i}$ 均为常数.

为了确定逆温层厚度, 通常采用理查孙判据确定法 ${ }^{[4]}$. 而事实上逆温基底的抬升过程是 原有逆温层不断耗散掉,而又在新的高度重新建立的过程. 此外,当混合层的气体进人逆温层 后, 如不再返回, 它的运动状态将发生根本的转变 ${ }^{[0]}$. 再考虑到重力波的形成、破碎及其与湍 流的相互作用等因素, 使我们不难得出结论: 抬升过程中逆温层的湍流结构是不稳定的, 采用 理查孙判据在理论和应用上都存在一些问题.

我们从 (10) 式出发,讨论逆温层的厚度变化,略去显含 $B$ 的项将 (10) 式改写成:

$$
\left[\frac{d h}{d t}-W(h)\right] \Delta \Theta=-[1-f(z, t)] \overline{(u \cdot \theta)_{h}},
$$

此处

$$
f(z, t)=\left[\frac{d}{d t}\left(\frac{1}{2} \Delta \Theta+\Theta_{m}\right) \Delta h-\Theta_{h+\Delta h} \frac{d \Delta h}{d t}\right] \overline{(w \theta)_{n}^{-2}} .
$$

观测表明, $\Delta h$ 随时间递增,若混合层外的流场具有稳定层结,则分析表明

$$
\frac{d}{d t}\left(\frac{1}{2} \Delta \Theta+\Theta_{m}\right) \Delta h-\Theta_{h+\Delta h} \frac{d \Delta h}{d t}>0 .
$$

于是 (21) 式给出

$$
\left[\frac{d h}{d t}-W(h)\right] \Delta \Theta>-\overline{(w \theta}_{h} .
$$

由此, 并根据 Yamada ${ }^{[11]}$ 的结果, 不难看出 $|f(z, t)|$ 是一个很小的数, 且它的变化对 (21) 式 的平衡只有较弱的影响。于是可以假设

$$
\left[\frac{d h}{d t}-W(h)\right] \Delta \Theta=-K \overline{(v \theta)_{h}},
$$

式中 $K$ 为经验常数,显然, $K>1$.

让 $B=0$, 将 (22) 式代人 (10) 式, 并考虑到在混合层发展的大部分时间内背景场层结 较弱, 可以得到下列方程

$$
\Delta \Theta \frac{d \Delta h}{d t}-\Delta h \frac{\partial \Theta_{m}}{\partial t}-2(K-1) \overline{(w \theta)_{h}}=0 .
$$

观测表明, 当混合层有了一定发展, $\frac{\Delta h}{h}$ 约在 $10 \%$ 到 $20 \%$ 之间. 取 $\frac{\Delta h}{h} \sim 0.15$, 并取 $K \sim$ $1.4, \sqrt{1-2 C_{t b}} \sim 0.15$, 并利用 (7), (18) 和 (22) 式得到 (23) 式左边各项的权重依序为 $+0.2,-1.0,+0.8$. 因而作为一级近似可以认为 (23) 式左边后两项基本平衡, 即

$$
\Delta h \frac{\partial \Theta_{m}}{\partial t} \simeq-2(K-1) \overline{(w \theta)_{h}} .
$$

汀是

$$
\begin{aligned}
& \Delta h=C_{\Delta h} h, \\
& \frac{d \Delta h}{d t}=C_{\Delta h} \frac{d h}{d t},
\end{aligned}
$$

其中 $C_{\Delta h}=\frac{2(K-1) C}{1+C}, C=\sqrt{1-2 C_{b b}}$. 
直接积分 (23) 式,并利用 (18) 式有

$$
\Delta h\left(t_{1}\right)=2(K-1) \int_{t_{0}}^{t_{1}} \frac{\overline{(w \theta)_{h}}}{\Delta \Theta} d t+\Delta h\left(t_{0}\right) \exp \int_{t_{0}}^{t_{1}} \frac{1+C}{C} \times \frac{-\sqrt{(w \theta)_{h}}}{h \Delta \Theta} d t .
$$

利用 (22) 式,上式可写成:

$$
\Delta h\left(t_{1}\right)=\frac{2(1-K)}{K}\left[h\left(t_{1}\right)-h\left(t_{0}\right)\right]+\Delta h\left(t_{0}\right) \exp \int_{t_{0}}^{t_{1}} \frac{1+C}{C} \times \frac{-\overline{(u \theta)_{h}}}{h \Delta \Theta} d t .
$$

从 (27) 式可看出, $\Delta h\left(t_{1}\right)$ 由两部分组成,其中

$$
\frac{2(1-K)}{K}\left[h\left(t_{1}\right)-h\left(t_{0}\right)\right] \leqslant 0,
$$

它反映了湍流混合将耗散逆温层这一事实. 而另一部分

$$
\Delta h\left(t_{0}\right) \exp \int_{t_{0}}^{t_{t}} \frac{1+C}{C} \times \frac{-\overline{(w \theta)_{h}}}{h \Delta \Theta} d t
$$

按指数规律增扣. 它表示由混合层进入逆温层的部分能量使逆温层的厚度有所发展. 一般说 来

$$
\exp \int_{t_{0}}^{t_{1}} \frac{1+C}{C} \times \frac{-\overline{\left(u^{\prime} g\right)_{h}}}{h \Delta \Theta} d t-\frac{2(K-1)}{K} \frac{\left[h\left(t_{1}\right)-h\left(t_{0}\right)\right]}{\Delta h\left(t_{0}\right)}>1,
$$

即 $\Delta h$ 将随时间而增大.

为了说明逆温层的热力学特性, 考察方程 (11), 略去大尺度速度散度引起的项, 有

$$
\frac{d \Delta \vartheta}{d t}=\left[\frac{d h}{d t}+\frac{d \Delta h}{d t}\right] \Gamma(h+\Delta h, t)-\left[\overline{(w \ni)_{3}}-\overline{(w \vartheta)_{h}}\right] h^{-1} .
$$

混合层中由于对流活动产生向上运动的气块不断浸蚀逆温层的下边界, 这将减小逆温层厚度, 增加逆温强度; 逆温层内由内波的破碎所形成的湍涡, 混合层中的空气进入逆温层以及逆温层 顶部湍流的影响等因素有增加逆温层厚度减小其强度的趋势。（27）和（28）两式对这两种作 用的平衡给出了定量的描述.

从上述讨论可知, 如果由地面增热引起的对流活动抬升了逆温层底部, 而上层空气稳定层 结较弱, 那么 $\Delta \Theta$ 趋于变小. 但这时 $\frac{d h}{d t}$ 趋于增加, 而 $\Delta h$ 随着混合层的发展也趋于增加, 于 是 (28) 式表明 $\Delta \Theta$ 有回升的趋势。只有当在足够长的时间内, 不等式 $\left[\frac{d h}{d t}+\frac{d \Delta t}{d t}\right] \Gamma<[\overline{(\boldsymbol{w} \bar{\theta}) \text { 。 }}$ 一 $\left.\widetilde{(w \theta)_{h}}\right] h^{-1}$ 都得到满足时, $\Delta \Theta$ 才有明显减小。如果这种情况能维持较长时间,逆温层将趋 于消失.一般这可能发生在当地时间中午左右. 如果这时逆温层还没有完全消失,中午以后 $\left[\overline{(w \theta)_{0}}-\overline{(w \theta)_{h}}\right] h^{-1}$ 将明显减小, 但由于较上层空气的稳定层结较弱, 在中午后 $1-2$ 小时内 2 $\frac{d h}{d t}$ 不会有明显减小, 于是 (28) 表明 $\Delta \theta$ 可能又有回升. 以上讨论对覆盖逆温层的抬开与维持 给予了理论上的说明.

\section{三、计算及其与观测结果的比较和几个推论}

方程(7)一(11)和 (20) 是一组描述混合层发展过程的封闭方程组. 对它们可作数值计算. 
为求解析解, 可采用由 (8)，(9)，(11)，(18)，(22) 式和 (25) 式构成的方程组. 从它们不难 导话下列方程

$$
\frac{d \Delta \theta}{d(\Gamma h)}+\frac{1+C}{K C} \times \frac{\Delta \theta}{\Gamma h}-\frac{2(K-1) C}{1+C} \Gamma \underset{d(\Gamma h)}{d h}-1=0 .
$$

通常大尺度下沉速度影响很小,上式左边第三项约为

$$
\frac{2(K-1) C}{1+C} \Gamma \frac{d h}{d(\Gamma h)} \simeq \frac{2(K-1) C}{1+C} \text {. }
$$

特它代人 (29) 式,所得方程满足条件 $h=0$ 时, $\Delta \theta=0$ 的解是

$$
\Delta \theta=C_{\Delta \theta} \Gamma h,
$$

其中

$$
C_{\Delta \theta}=\frac{[1+(2 K-1) C] K C}{(1+C)[1+(K+1) C]} .
$$

由(24)和 (30) 式得到逆温强度

$$
\frac{\Delta \theta}{\Delta h}=\frac{C_{\Delta \theta}}{C_{\Delta h}} \Gamma .
$$

若没有剧烈的天气过程, 如高空强大的冷空气下沉引起下沉逆温 ${ }^{[14]}$, 则 (31) 式使得有可能根 据每天早晨的气球探测资料粗略估计 1 -2 公里内大气层中的局地逆温强度. 将 (30) 式代入 (11)式,并积分,给出混合层高度的变化

$$
\begin{aligned}
h^{2}(t)= & \frac{2(1+C)}{\Gamma(0)\left(1-C_{\Delta \theta}+C_{\Delta h}\right)}\left[\exp \frac{-2\left(1-C_{\Delta A}\right)}{1-C_{\Delta \theta}+C_{\Delta h}} B t\right] \cdot \int^{t}\left[\exp \frac{1-C_{\Delta \theta}-C_{\Delta h}}{1-C_{\Delta \theta}+C_{\Delta h}} B \tau\right] \\
& \cdot\left(\overline{\left.u^{\prime}\right)_{0}} d \tau+C_{h} \exp \frac{-2\left(1-C_{\Delta \theta}\right)}{1-C_{\Delta \theta}+C_{\Delta h}} B t,\right.
\end{aligned}
$$

式中 $C_{h}$ 是积分常数.

下面给出几个重要推论。

1) 考虑逆温层中的能耗散. 令 $B=0$,由(32) 式,有

$$
\frac{d h^{2}}{d t} \propto \frac{\overline{(\omega \theta)_{0}}}{\Gamma} \text {. }
$$

用积分类比代替 $\frac{d h^{2}}{d t}$, 并将 $u_{f}^{3}=\frac{g}{\Theta} \overline{(u \theta)_{i}} h, \frac{h}{t} \propto u_{f}$ 代人上式,给出

$$
\varepsilon_{i i}=C_{i} \frac{u_{f}^{3}}{\Delta h}=C_{i} u_{j}^{2} N,
$$

式中 $N=\left[\frac{g}{\Theta} \Gamma\right]^{\frac{1}{2}}$ 是浮力频率. 于是,能耗散由湍流量 $u_{f}^{2}$ 与背景场特征量 $N$ 的相互作用表示 出来. 而且,它的波动形式也说明在导出 (20) 式时，将内波辐射引起的能耗散纳人 $u_{f}^{3} / \Delta h$ 是 合理的.

2) 若 $\overline{(w 9)_{0}} \equiv 0$, 则混合层发展完全由动力作用所引起. 此时, 由(31)式可求得相应的 逆温强度 $\frac{\Delta \theta_{*}}{\Delta h}$ 为: 


$$
\frac{\Delta \Theta_{*}}{\Delta h}=\frac{(2 K-1) K}{2\left(K^{2}-1\right)} \Gamma
$$

此外,与 (22) 式相类似的有

$$
\Delta \Theta_{*} \frac{d h}{d t}=-K \overline{(w \theta)_{h}} .
$$

由 (19)，(34) 和 (35) 式, 可得到

$$
\frac{d h}{d t}=\frac{4\left(1-C_{t m}\right)\left(K^{2}-1\right)}{2 K-1} \frac{u_{*}^{2} U_{m}}{\Delta h h N^{2}} .
$$

上式可写成

$$
\frac{d h}{d t}=C_{*} \frac{u_{*}^{3}}{\Delta h h N^{2}}=C_{*} \frac{u_{*}}{\xi_{1} \xi_{3}},
$$

其中 $C_{*}$ 是常数,而

$$
\xi_{1}=\underset{u_{*}}{h N}, \quad \xi_{2}=\frac{\Delta h N}{u_{*}} .
$$

Tennekes $^{[3]}$ 在类似情况下得到的公式是:

$$
\frac{d h}{d t}=C \frac{u_{*}^{3}}{h^{2} N^{2}},
$$

其中 $C$ 是常数.

如果不考虑大尺度下沉速度，减小混合层发展速率的主要因索是由重力波引起的能量损 失. 于是 (37) 式表明: 由波辐射引起的能耗散分别与无量纲参数 $\xi_{1}$ 和 $\xi_{2}$ 成比例.

显然， $\xi_{1}$ 所描述的是与混合层高度等量级的大尺度浴的活动有关的、压力脉动所产生的 波动现象. 而 $\xi_{2}$ 除了描述较小波长(与 $\Delta h$ 等量级)的重力波活动外,注意到

$$
\xi_{2}=\frac{\Delta h N}{u_{*}}=\frac{u_{*}^{2} N}{u_{*}^{3} / \Delta h},
$$

它给出逆温层内波与湍流的相互影响. 若 $u_{*}^{2} N \gg \frac{u_{*}^{3}}{\Delta h}$, 则从混合层输送上来的能量大部分被 波辐射损失掉,即能量收支方程中的 $-\frac{\partial}{\partial z} \overline{[e w]}$ 与 $-\frac{\partial}{\partial z}\left[\frac{1}{\rho} \overline{p w}\right]$ 基本平衡, 因而逆温层

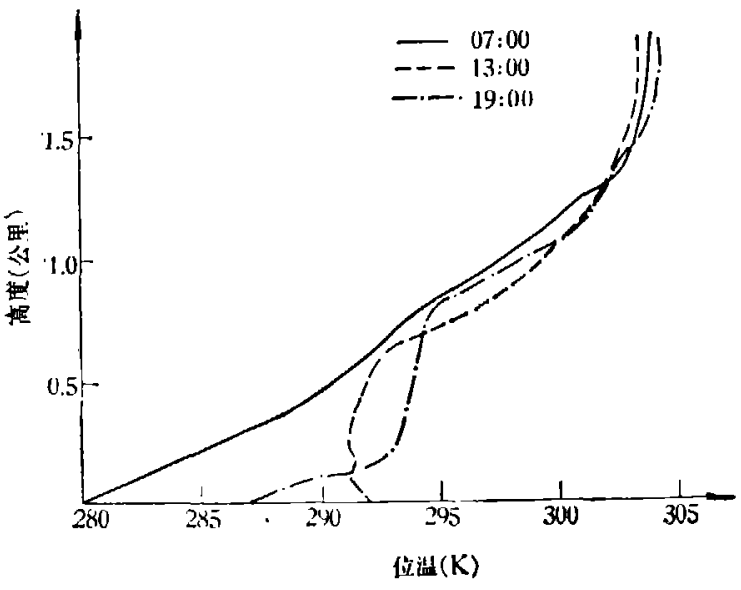

图 1 - 位温邡线（例 1)

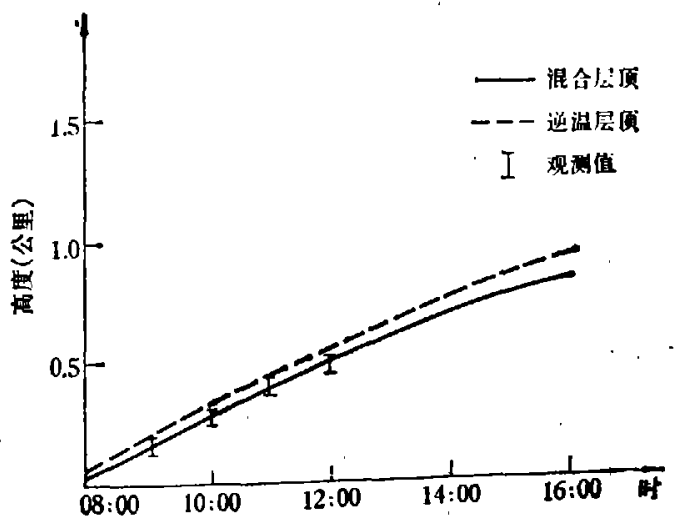

图 2 混合层高度与逆温层厚度的变化（1）。 
的局地湍能耗散速率很小,这意味着 $\frac{d h}{d t}$ 减小。

现在给出两个计算实例。在计算中,取 $B=0, K=1.4$.

例 1. 1977 年 10 月 12 日，声雷达设置在北京西郊机场。地面热通量是根据地面梯度观 测, 并应用文献 [12] 的方法计算的. 这天地面热通量变化不规则, 其最大值为 $0.25^{\circ} \mathrm{K} \cdot$ 米/秒. 图 1 是根据北京气象台的探空资料绘制的位温廓线, 07:00 时的位温廓线作为理论计算时的 初始值. 图 2 给出混合层高度与逆温层厚度的变化, 图中的观测值是从声雷达照片中得出的.

例 2. Wangara 实验第 32 天 ${ }^{[13]}$. 地面热通量可表示为:

$$
\overline{(w \theta)_{0}}=\overline{(w \theta)_{0 m: x}} \cos u^{\prime}\left(t_{0}-t\right),
$$

式中 $t_{0}$ 是当地中午时间, $\overline{(u \cdot \theta)_{0 \text { max }}}=0.157^{\circ} \mathrm{K} \cdot /$ 米秒(热通量数值出于文献[11]). 图 3 是根 据观测资料绘制的位温廓线。图 4 是混合层高度随时间的变化，其中观测值是从位温廓线中 得出. 由于 Wangara 观测资料的限制, 在计算的时段内只给出 12:04 和 15:00 的混合层高度 观测值.

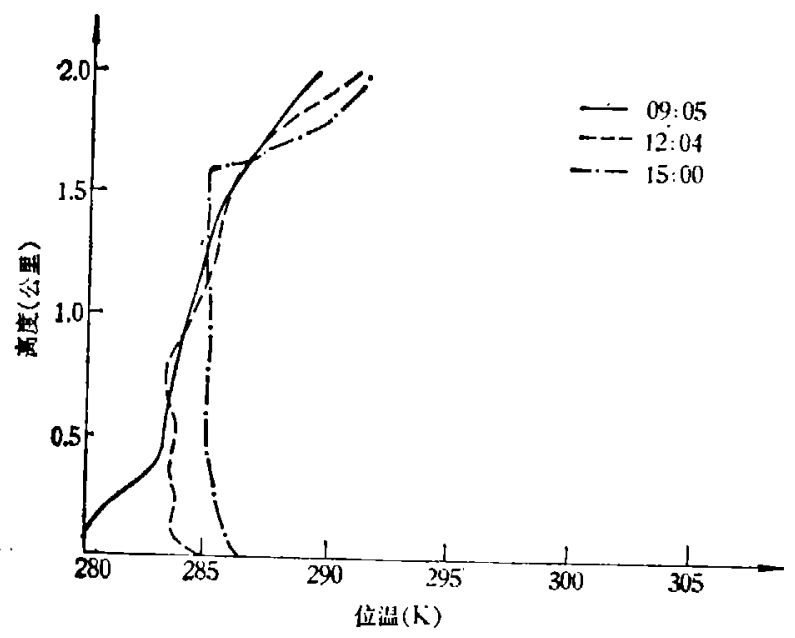

图 3 位温廊线 (例 2)

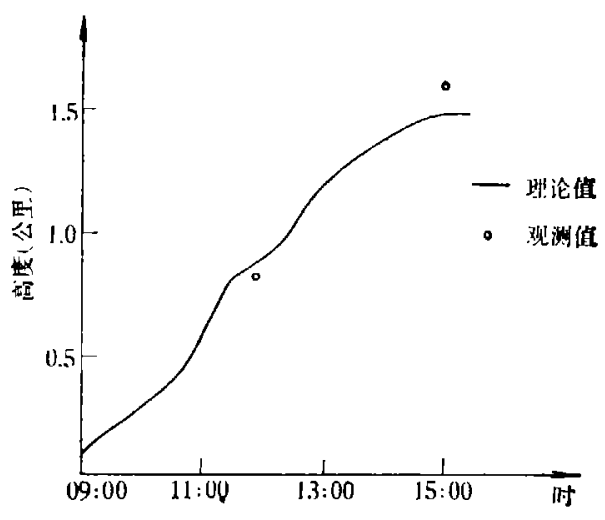

图 4 混合层高度的变化 (例 2)

\section{四、结 束 语}

由以上讨论可以得到下面的结果。

在晴朗小风天气条件下, 日出后, 混合层的形成、逆温层抬升的物理过程是相当复杂的。我 们讨论了这一物理过程的能量收支问题, 对混合层顶的覆盖逆温层的形成和维持给予理论上 的说明.

在此基础上,获得了描写混合层高度、逆温层厚度及其逆温强度的方程组. 理论结果与观 测实例的结果基本一致。

由于较合理的利用了三层模式的性能，使得能对混合层发展过程中的复杂的物理现象作 进一步的讨论,并建立了较合适的参数化方程。通过修正跳跃模式的基本方程,保持了两者的 
争机咲系, 四而此模式可以对大气边哭层中基本金引过程的讨论进一步深化.

\section{参考文 献}

[l ] Liı!l. 1. K., Quarl. J. Roy. Metcor. Sor. 86 (1960), 483-494.

[Q] Carson, D. J., ibid., 99 (1973), 450-467.

[3] Tennekes, H., J. Atmos. Sci., 30 (1973), $553-567$.

[4] Mahrt. L. \& Lenschow, D. H., ibid., 33 (1976), 41-51.

[J] Deardorff, J. W., et al., J. Fluid., Mech., 35 (1969), 7-31.

[6] — Bacndary-Layer Meteor., 7(1974). 199-_60.

[ 7 ] 吕乃平等,环境科学, $\mathbf{1}(1980), 44-49$.

[8] Stull, R. B., J. Atmos. Sci., 33(1976). 1268-1278.

[9] Panofsky, H. A., Boundary-Layer Metcor., 4 (1973), 251-264.

[10] Busch, N. E., ibid., 4(1973), 213-240.

[11] Yamada, T. \& Berman, S., J. Appl. Meteor., 1979, Junc, 781-786.

[12] Monin, A. S. \& Yaglom, A. M., Statistical Fluid Mechanics, Cambridge, MIT Press.

[13] Clarke, et al., Tech. Paper, No. 19, Div. Meteor. Phys, CSIho Mclbourne, Australia

[14] Ming-yu Zhou, et al,, J. Acoust. Soc. Am., 1981, 68(1). 